\title{
Implementación de un cuestionario de calidad de vida relacionada con la salud para niños y adolescentes con enfermedad celíaca
} Implementation of a health related quality of life questionnaire for children and adolescents with celiac disease

\author{
Lic. Mercedes Pico y Lic. María Florencia Spirito ${ }^{a}$
}

\section{RESUMEN}

Introducción. En los pacientes con enfermedad celíaca, medir la calidad de vida relacionada con la salud permite un abordaje integral.

Objetivo. Evaluar la implementación y la sensibilidad al cambio de la versión argentina del cuestionario específico CDDUX para medir la calidad de vida relacionada con la salud en niños y adolescentes.

Métodos. Estudio descriptivo, observacional, longitudinal y prospectivo. Participaron niños y adolescentes de 8 a 18 años con enfermedad celíaca, su padre o tutor, y un gastroenterólogo. La calidad de vida relacionada con la salud se evaluó con el CDDUX y la sensibilidad al cambio, mediante la variación de su puntaje aplicado en dos oportunidades y una escala de percepción del cambio desde la opinión del paciente y su padre. También la experiencia en su implementación con un formulario sobre fortalezas y debilidades completado por pacientes, padres y gastroenterólogos.

Resultados. En la primera entrevista 118 binomios niño/padre completaron el CDDUXy registraron la experiencia; 102 lo completaron en dos oportunidades. Los puntajes totales fueron más elevados en la segunda $(p=0,009$ y 0,003 , respectivamente). Hubo congruencia entre la percepción de cambio expresada por el niño y por el padre, y la diferencia en el puntaje del CDDUX entre consultas $(p<0,001)$. Tamaño del efecto estandarizado: 0,52 en el caso de los niños y 0,45 en los padres. Hubo acuerdo del $64 \%$ en las opiniones de los niños con los padres (kappa 0,20) y del $51 \%$ con los médicos (kappa 0,10 ) respecto del cambio entre consultas (mejor-igual-peor). Los gastroenterólogos también registraron su experiencia con el CDDUX. Hubo consenso en que fue satisfactoria y de utilidad.

Licenciadas en Nutrición. Hospital de Pediatría "Prof. Dr. Juan P. Garrahan".

Correspondencia: Lic. Mercedes Pico: mercedespico@yahoo. com.ar

Conflicto de intereses: Ninguno que declarar. Conclusiones. El CDDUX demostró estabilidad test-retesty sensibilidad al cambio. Hubo visiones diferentes respecto del cambio en la calidad de vida relacionada con la salud del niño, con mayor acuerdo entre los niños y los padres que con el médico. Este cuestionario es aceptado por los niños, los padres y los gastroenterólogos.

Palabras clave: calidad de vida, niños, enfermedad celíaca, CDDUX, sensibilidad al cambio.

http:/ /dx.doi.org/10.5546/aap.2014.e19

\section{INTRODUCCIÓN}

La importancia de incluir a los niños como sujetos, cuyas perspectivas sean escuchadas y valoradas en la consulta, está ganando impulso en la investigación infantil. Hay un cambio de paradigma, con un enfoque en la vida cotidiana de los niños desde su perspectiva, que ahora tienen nuevos roles en el proceso de medición y seguimiento de su bienestar. ${ }^{1,2}$

La calidad de vida relacionada con la salud (CVRS) es el indicador de uso más frecuente para evaluar el nivel de bienestar de las personas respecto de su salud. Shumaker y Naughton la definieron como "...la evaluación subjetiva de la influencia del estado de salud, los cuidados sanitarios y la promoción de la salud, sobre la capacidad del individuo para mantener un nivel de funcionamiento que le permite realizar las actividades que le son importantes, y que afectan su estado general de bienestar...". Esta definición parte de la percepción que tiene el individuo sobre su propia salud; también es subjetiva y multidimensional. ${ }^{3,4}$

En las enfermedades crónicas, su medición brinda información complementaria para la toma de decisiones en la práctica médica y es útil para detectar situaciones que no hubieran surgido en la modalidad de atención tradicional, lo que permite mejorar la asistencia o plantear diferentes estrategias para optimizar el cumplimiento terapéutico. ${ }^{5-7}$

En una revisión sistemática de instrumentos de CVRS en pediatría, se encontró que la mayoría presentaron resultados aceptables de confiabilidad 
y validez, siendo pocos los que evalúan su estabilidad test-retest y la sensibilidad al cambio. La estabilidad test-retest mide el grado de repetibilidad de las respuestas de un mismo individuo recogidas en dos o más ocasiones, cuando supuestamente no ha habido cambios en su estado de salud. ${ }^{8}$ La sensibilidad al cambio de un instrumento es la capacidad que este tiene de detectar, a través de las modificaciones en sus puntajes, cambios en la calidad de vida de las personas cuando estos han tenido lugar. ${ }^{9}$

Por eso, resulta de interés fomentar los estudios longitudinales o los ensayos clínicos en los que se utilicen los instrumentos de la CVRS para el seguimiento de los pacientes en el tiempo. ${ }^{10}$

Implementar un instrumento para medir la CVRS en la práctica clínica requiere una evaluación del proceso para poder detectar situaciones que puedan interferir en su utilización y elaborar estrategias de abordaje. También es importante establecer si, luego de aplicado, puede detectar los cambios que se producen en el seguimiento de los pacientes.

La enfermedad celíaca (EC) es un trastorno cuya afectación física se resuelve a poco de iniciar el tratamiento dietoterápico; entonces, la valoración de los aspectos psicosociales cobra gran relevancia. La dieta, que debe implementarse de por vida, implica restricciones y alteraciones en la vida social del niño que pueden afectar su calidad de vida y repercutir en el cumplimiento del tratamiento. ${ }^{11-15}$

Con el objetivo de contar con una herramienta que permita abordar la problemática de la CVRS de niños y adolescentes con EC hemos adaptado y validado, de acuerdo con los lineamientos internacionales, el cuestionario especifico CDDUX, que mostró adecuada validez y confiabilidad, y que demanda poco tiempo de realización: en promedio, 4 minutos. ${ }^{16-19}$

El objetivo de esta investigación fue evaluar la implementación y la sensibilidad al cambio de la versión argentina del cuestionario específico CDDUX.

\section{Hipótesis}

- Es factible implementar en la práctica asistencial el cuestionario CDDUX.

- La percepción de los cambios observados por los pacientes y sus padres entre dos consultas se refleja en los valores de puntaje del CDDUX y demuestra que es capaz, en forma preliminar, de tener sensibilidad al cambio.

\section{POBLACIÓN Y MÉTODOS}

Estudio descriptivo, observacional, longitudinal y prospectivo realizado en el Hospital de Pediatría "Prof. Dr. Juan P. Garrahan", entre el 1 de julio de 2010 y el 30 de junio de 2011, en el marco del consultorio interdisciplinario de atención de enfermedad celíaca en el que trabajan, en conjunto, gastroenterólogos pediatras y nutricionistas.

Se incluyeron niños y adolescentes de 8 a 18 años con diagnóstico de enfermedad celíaca definida por biopsia de intestino, que estuvieran realizando el tratamiento por un tiempo mayor de 6 meses y que no presentaran intercurrencias agudas que afectaran su salud.

Se excluyó al niño/adolescente o padre que no pudiera comprender el cuestionario y formulario, y al niño/adolescente con otra patología crónica que requiriera otras restricciones dietarias. El reclutamiento se hizo en forma consecutiva.

La estimación de la muestra estuvo en relación con la factibilidad de su obtención en el marco de la población asistida.

Participaron gastroenterólogos integrantes del equipo de atención de enfermedad celíaca, quienes tuvieron una capacitación previa sobre la comprensión del cuestionario CDDUX y su interpretación. Consideraciones éticas: se solicitó el consentimiento informado firmado por el padre o tutor, y el asentimiento del niño/adolescente para participar.

El protocolo fue aprobado el 4 de junio de 2010 por el Comité de Ética de Investigación del Hospital "Prof. Dr. Juan P. Garrahan".

\section{Variables principales de resultado}

Calidad de vida relacionada con la salud: medida con el CDDUX. El instrumento cuenta con 12 ítems agrupados en tres subescalas: "Tener enfermedad celíaca" (3 ítems), "Comunicación" (3) y "Dieta" (6). Valorada con una escala de 1 a 100, codificada con una escala de Lickert de 5 puntos. Los valores mayores indican mejor calidad de vida (rango 0 a 100; 1 a 20: muy mala, 21 a 40: mala, 41 a 60: neutra, 61 a 80: buena, 81 a 100: muy buena). El puntaje total surge de la suma de los puntajes de cada ítem dividido el número de ítems.

El CDDUX fue completado por los niños y los padres antes de ser atendidos por el gastroenterólogo y los resultados se le comunicaron a este como un dato complementario de la consulta. Al finalizar la primera consulta, se registró si se in- 
dicó alguna intervención terapéutica a partir de los resultados del CDDUX y lo observado (derivación a otros profesionales del equipo interdisciplinario, nutricionista, psicóloga, médica clínica, participación de reuniones grupales con otros pacientes celíacos, etc.).

Sensibilidad al cambio: valorada mediante la variación del puntaje obtenido en el CDDUX administrado en dos oportunidades (durante el seguimiento ambulatorio y con un intervalo mínimo de 3 meses). Para evaluar si esta variación reflejaba los cambios ocurridos se utilizó una escala de precepción de cambio.

Percepción del cambio: se exploró mediante una escala cerrada categorizada en: mejor-igualpeor en respuesta a la pregunta sobre cómo consideraban la calidad de vida del niño (según aspectos evaluados en el CDDUX) en relación con la consulta anterior. Esta escala fue completada por los niños, los padres y los gastroenterólogos en la segunda consulta, para evaluar el acuerdo entre opiniones.

Experiencia en la administración del CDDUX: medida con un formulario de recolección de datos, de elaboración propia, con preguntas cerradas de respuesta sí-no y una pregunta abierta sobre las dificultades y fortalezas en su implementación, con el fin de recoger información sobre el uso del CDDUX desde el punto de vista del niño/adolescente, padre o tutor, y gastroenterólogo. En la primera consulta, los niños y sus padres respondieron luego de completar el CDDUX; el gastroenterólogo, al finalizar la consulta.

\section{Variables secundarias}

- Edad del paciente en años cumplidos.

- Sexo.

- Situación socioeconómica (no pobre-pobreindigente): según el acceso a la canasta básica familiar del INDEC.

- Condición actual de la enfermedad (asintomático o no): asintomático: cuando no presentaba signos clínicos ni síntomas físicos característicos de la condición.

- Cumplimiento de la dieta sin gluten (cumplimiento o no): cumplimiento: si no se detectaban transgresiones en el interrogatorio efectuado por la licenciada en Nutrición, con 5 años de experiencia en esta patología, y por los datos de laboratorio (anticuerpo antitransglutaminasa negativo realizado en un tiempo menor de 6 meses).

\section{Análisis estadístico}

Los resultados de las opiniones de los gastroenterólogos y las familias se expresan como porcentajes de respuesta, y los puntajes del CDDUX, como valor medio y desviación estándar.

Se evaluó la presencia y dirección de cambios en la media de los puntajes totales del CDDUX mediante la prueba de la $t$ para muestras pareadas entre los diferentes momentos de aplicación y, a su vez, con la escala de cambio percibido por el paciente y su padre.

Para evaluar la sensibilidad al cambio se consideró el tamaño del efecto estandarizado (SES: delta del puntaje/desviación estándar del puntaje basal), tomando en consideración un SES mayor de 0,20 como relevante..$^{19,20}$

Además, se exploró la concordancia entre la opinión de los niños con sus padres y de los niños con su médicos mediante el estadístico kappa.

Retrospectivamente, de acuerdo con los datos obtenidos, podemos inferir que el estudio tuvo un poder del $80 \%$ (error beta 0,20 ) para determinar una diferencia entre el valor basal del CDDUX y el obtenido en la segunda medición.

Para los análisis se consideró significativo un valor de $p<0,05$.

Se utilizó el programa estadístico SPSS 15.0 para Windows.

\section{RESULTADOS}

Se incluyeron 118 binomios niño/adolescentepadre en la evaluación de la experiencia en la implementación del cuestionario, ya que completaron el formulario $\mathrm{y}$, a su vez, realizaron el CDDUX. De este grupo, 102 binomios niño/ adolescente-padre completaron por segunda vez el CDDUX y la escala de precepción de cambio en una consulta posterior, quedando conformada la muestra para evaluar la sensibilidad al cambio. La pérdida de 16 pacientes se debió a la inasistencia a la consulta posterior durante el tiempo que duró la investigación. No hubo rechazos a participar.

Participaron cinco gastroenterólogos integrantes del equipo interdisciplinario, tres de ellos de la planta permanente y dos residentes.

Para evaluar la sensibilidad al cambio del cuestionario se analizaron 102 casos que completaron el CDDUX en dos oportunidades, pudiendo explorar si había modificaciones en el puntaje obtenido y si estas se correlacionaban con la percepción de cambio del propio niño y de su padre/tutor. 
De los 102 niños, 39,2\% $(n=40)$ eran varones y $60,8 \%(n=62)$, mujeres. Las características clínicas y socioeconómicas se muestran en la Tabla 1. En la primera administración del cuestionario solo dos niños presentaron algún síntoma digestivo y todos se encontraban asintomáticos en la segunda administración. El 73\% al 77\% refirieron cumplir la dieta sin gluten.

El tiempo medio entre la primera y la segunda administración fue de 9 meses, con un mínimo de 3 y un máximo de 21 meses.

El puntaje total del CDDUX realizado por niños/adolescentes y padres fue más elevado en la segunda administración al compararlo con la primera y alcanzó significación estadística en ambos grupos (Tabla 2).

De los 102 niños, en un 32,4\% $(n=33)$ los resultados del CDDUX en la primera administración ameritaron alguna intervención y este grupo tuvo puntajes globalmente más bajos que quienes no la requirieron: 58,93 (DE 11,05) contra 71,03 (DE 13,45); en la segunda administración, los puntajes fueron 65,34 (DE 12,99) y 73,05 (DE 13,13) respectivamente. La variación promedio del puntaje fue de 6,41 puntos y de 3,01 en los grupos con y sin intervención.

Se observó congruencia entre la percepción de cambio expresada por el niño y el padre, y la dife- rencia en el puntaje del CDDUX entre la primera y la segunda consulta; los resultados fueron significativos en quienes manifestaron cambio.

El tamaño del efecto estandarizado fue de 0,52 en el caso de los niños y de 0,45 en los padres (Tabla 3).

Cabe destacar que en un solo caso hubo un informe de "estar peor", con una diferencia en el puntaje medio del CDDUX del niño de -16,66.

En la Figura 1 se presenta la frecuencia en la que padres, niños y médicos percibieron el cambio de condición del niño (está mejor, igual o peor) entre las consultas; la mayoría de los niños y de los padres tuvieron una percepción de mejoría, mientras que la mayoría de los gastroenterólogos los veía igual.

$\mathrm{Al}$ analizar la concordancia entre las opiniones de niños con padres y de niños con médicos, hubo acuerdo del $64 \%$ con los padres (kappa 0,20 ) y aun menor $(51 \%)$ con sus médicos (kappa 0,10$)$.

En las Tablas 4 y 5 se muestran las opiniones de los niños, los padres y los gastroenterólogos. La mayoría de los niños y los padres acordaron en una opinión satisfactoria y de utilidad, ya que consideraron que puede ayudar a expresar cosas que no hubieran sido escuchadas en la consulta y que es importante que el gastroenterólogo conozca. Asimismo, la mitad de los entrevistados

TABLA 1. Características clínicas y socioeconómicas de los pacientes en la primera y la segunda administración del cuestionario CDDUX

\begin{tabular}{|c|c|c|c|c|}
\hline Características & & Primera administración & Segunda administración & Valor de $p$ \\
\hline $\begin{array}{l}\text { Condición actual } \\
\text { de la enfermedad }\end{array}$ & Asintomático & $99 \%(n=100)$ & $100 \%(n=102)$ & 0,57 \\
\hline $\begin{array}{l}\text { Cumplimiento de } \\
\text { la dieta sin gluten }\end{array}$ & Sí & $73,3 \%(\mathrm{n}=74)$ & $77,5 \%(\mathrm{n}=79)$ & 0,40 \\
\hline Nivel socioeconómico* & $\begin{array}{c}\text { No pobre } \\
\text { Pobre /indigente }\end{array}$ & $\begin{array}{l}77,5 \%(n=79) \\
22,6 \%(n=23)\end{array}$ & $\begin{array}{l}82 \%(n=82) \\
18 \%(n=18)\end{array}$ & $\begin{array}{l}0,47 \\
0,38\end{array}$ \\
\hline
\end{tabular}

* Línea de pobreza según el acceso a la canasta básica familiar por cálculo del INDEC.

TABLA 2. Valor medio y desviación estándar para el puntaje total del cuestionario CDDUX según los niños y los padres en la primera y la segunda administración

\begin{tabular}{|c|c|c|c|c|}
\hline & $\begin{array}{l}\text { Primera administración } \\
\text { (102) }\end{array}$ & $\begin{array}{c}\text { Segunda administración } \\
\text { (102) }\end{array}$ & $\begin{array}{l}\text { Diferencia } \\
\text { X (IC 95\%) }\end{array}$ & Valor de $p$ \\
\hline $\begin{array}{l}\text { Puntaje total niños } \\
X(D E)\end{array}$ & $67,12(14)$ & $71,23(13,65)$ & $+4,11$ & 0,009 \\
\hline $\begin{array}{l}\text { Puntaje total padres } \\
\text { X (DE) }\end{array}$ & $60,63(13,6)$ & $64,44(13,56)$ & $+3,80$ & 0,003 \\
\hline
\end{tabular}


Figura 1. Cambio en la condición del niño (mejor, igual o peor) entre la primera y la segunda visita según la percepción del niño, su padre y el gastroenterólogo interviniente en la atención

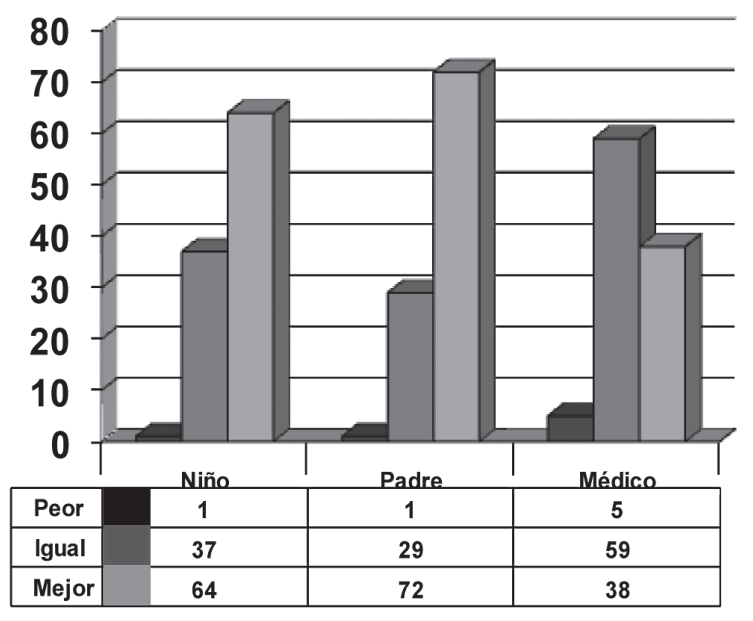

expresaron que completar el cuestionario les hizo pensar cosas que no habían pensado antes. Casi todos los gastroenterólogos consideraron que la realización del cuestionario, por parte de los pacientes, no entorpeció el circuito de atención ni afectó el desarrollo de la consulta; a su vez, acordaron que los resultados brindaron información importante y permitieron detectar aspectos que necesitaron alguna acción.

\section{DISCUSIÓN}

La versión argentina del CDDUX ha cumplido los pasos de adaptación transcultural y validación. Con esta investigación, hemos explorado su implementación y sensibilidad al cambio.

A pesar de que las características de los pacientes entre la primera y la segunda administración resultaron de gran similitud, hubo diferencias en los resultados del CDDUX; los puntajes fueron significativamente mayores en el segundo tiempo.

TABla 3. Presencia y dirección de los cambios en la media de los puntajes del CDDUX entre los diferentes momentos de aplicación, tomando como referencia la escala de cambio percibido por el niño y el padre/tutor

\begin{tabular}{lcccccc}
\hline Cuestionario & $\begin{array}{c}\text { Cambio de } \\
\text { percepción propia }\end{array}$ & $\begin{array}{c}\text { Primera aplicación } \\
\text { media (DE) }\end{array}$ & $\begin{array}{c}\text { Segunda aplicación } \\
\text { media (DE) }\end{array}$ & $\begin{array}{c}\text { Delta media de las } \\
\text { diferencias (DE) }\end{array}$ & $\begin{array}{c}\text { Valor } \\
\text { de } p^{*}\end{array}$ & $\begin{array}{c}\text { SES** } \\
\text { Puntaje }\end{array}$ \\
Sí & $67,89(13,45)$ & $74,91(12,59)$ & $7,02(14,67)$ & $<0,001$ & 0,52 \\
total niños & No & $65,76(15,02)$ & $64,77(13,17)$ & $-0,99(16,20)$ & 0,71 & \\
Puntaje total & Sí & $60,17(13,24)$ & $66,13(13,46)$ & $5,95(13,22)$ & $<0,001$ & 0,45 \\
padres & No & $61,77(14,65)$ & $60,16(13,09)$ & $-1,60(10,59)$ & 0,42 & \\
\hline
\end{tabular}

${ }^{*}$ Prueba de la $t$ pareada con alfa de 0,05 .

** Tamaño del efecto estandarizado mayor de 0,2.

TABLA 4. Resultados del formulario sobre implementación del cuestionario según niños/adolescentes y padre/tutor

\begin{tabular}{|c|c|c|c|c|}
\hline \multirow[t]{2}{*}{ Pregunta } & \multicolumn{2}{|c|}{ Niños $(n=117)$} & \multicolumn{2}{|c|}{ Padres $(n=118)$} \\
\hline & Sí (\%) & No $(\%)$ & Sí (\%) & No $(\%)$ \\
\hline $\begin{array}{l}\text { ¿Demandó mucho tiempo } \\
\text { contestar el cuestionario? }\end{array}$ & 17,1 & 82,9 & 9,3 & 90,7 \\
\hline ¿Resultó molesto haberlo hecho? & 2,6 & 97,4 & 1,7 & 98,3 \\
\hline $\begin{array}{l}\text { ¿Le hizo pensar en cosas } \\
\text { que no había pensado antes? }\end{array}$ & 52,1 & 47,9 & 57,3 & 42,7 \\
\hline $\begin{array}{l}\text { ¿Ayuda a expresar cosas que de } \\
\text { otra manera no le hubieran preguntado? }\end{array}$ & 71,8 & 28,2 & 85,6 & 14,4 \\
\hline $\begin{array}{l}\text { ¿Considera importante que el } \\
\text { médico sepa los aspectos que se } \\
\text { abordan en el cuestionario? }\end{array}$ & 94,9 & 5,1 & 96,6 & 3,4 \\
\hline
\end{tabular}


Tabla 5. Resultados del formulario sobre la implementación del cuestionario según el médico gastroenterólogo

\begin{tabular}{lcc}
\hline Pregunta & \multicolumn{2}{c}{ Gastroenterólogo (n= 118) } \\
& Sí (\%) & No (\%) \\
\hline ¿El cuestionario entorpeció el circuito de atención? & 6,8 & 93,2 \\
¿El cuestionario afectó el desarrollo de la consulta? & 2,5 & 97,5 \\
¿Los resultados brindaron información importante? & 96,6 & 3,4 \\
A partir de los resultados, ¿detectó aspectos que considera & & 44,1 \\
que necesitan alguna acción? & 55,9 & 0,8 \\
¿Acuerda con la inclusión sistemática del cuestionario en la consulta? & 99,2 & \\
\hline
\end{tabular}

Al analizar la propiedad del CDDUX de medir la sensibilidad al cambio, se observó que la modificación del puntaje pudo revelar los cambios en la condición del niño; el cuestionario demostró tener estabilidad test-retest (sin cambios en los puntajes en aquellos que referían ausencia de cambios en el período estudiado) y sensibilidad al cambio, con un tamaño del efecto estandarizado de 0,52 en el caso de los niños y 0,45 en los padres; y pueden considerarse relevantes, según el parámetro de evaluación de esta propiedad que tienen en cuenta autores como Pane y Solans en sus revisiones de los cuestionarios de calidad de vida relacionada con la salud..$^{10,20}$

Los resultados permiten sugerir que el instrumento puede detectar cambios en aspectos de la calidad de vida del niño celíaco que no se muestran a nivel clínico.

Los niños, padres y gastroenterólogos tuvieron percepciones diferentes. Existió mayor acuerdo entre los niños y los padres que entre los niños y los médicos, también observado en otras investigaciones que evaluaron estos aspectos..$^{21-23}$

Las diferencias en la percepción de la calidad de vida resaltan la importancia de considerar la propia perspectiva de los niños respecto de su salud, sin desconocer la de los padres, ya que aporta una visión complementaria. Los padres a menudo carecen de primera mano de información, por ejemplo, con respecto a la experiencia en la escuela o de las interacciones sociales de los niños. El no acuerdo entre padres e hijos puede ser capitalizado en la consulta como fuente de información para abordar posibles situaciones de conflicto o factores emocionales que no hubieran surgido. Esta discordancia se observó tanto en sujetos sanos como en aquellos con trastornos psiquiátricos, migrañas, enfermedad inflamatoria intestinal, dolor abdominal, artritis juvenil, cáncer, etc. ${ }^{24}$

Se ha argumentado que las discrepancias entre los niños y los informes de los padres/tutores podrían reflejar válidamente la perspectiva de cada encuestado y no solo inexactitud o sesgo. ${ }^{24}$

Por lo mencionado, y dados el escaso número de categorías y la prevalencia del fenómeno estudiado, los resultados de concordancia de opiniones entre padres, niños y médicos deben ser evaluados con cautela.

En el cuestionario CDDUX holandés, no se analizó la experiencia de implementación ni la sensibilidad al cambio; por lo tanto, nuestros resultados no se pudieron comparar con el cuestionario original.

Debido a que los pacientes participantes de este estudio eran de un nivel socioeconómico medio y bajo, los celíacos de mayores recursos no estuvieron representados, lo cual plantea un desafío para quienes trabajan con estos pacientes.

El CDDUX es un instrumento aceptado y considerado de utilidad por los niños, los padres y los médicos.

La aplicación de instrumentos como el CDDUX puede permitir a los profesionales de la salud conocer aspectos emocionales y de integración social que puedan estar afectando, por ejemplo, la adhesión al tratamiento y, a su vez, evaluar los cambios del paciente en el tiempo.

\section{CONCLUSIONES}

- Las características clínicas resultaron similares en diferentes consultas, pero se observaron diferencias en los valores medios del CDDUX.

- El cuestionario demostró tener estabilidad testretest y sensibilidad al cambio.

- Niños, padres y gastroenterólogos tuvieron visiones diferentes respecto del cambio en la calidad de vida del niño entre la primera y la segunda administración del CDDUX, con mayor acuerdo entre niños y padres que con el médico. 
- El CDDUX mostró ser un instrumento aceptado por niños, padres y gastroenterólogos.

\section{Agradecimientos}

A la Dra. Susana Rodríguez y a la Dra. Mariana Roizen, por su asesoría permanente y guía durante todo el proceso que duró la investigación.

A la Dra. Adriana Bottero y los médicos gastroenterólogos integrantes del Equipo Interdisciplinario de Atención de Enfermedad Celíaca, por su participación desinteresada y colaboración para con el desarrollo de la investigación.

\section{BIBLIOGRAFÍA}

1. Mosteiro Díaz P, Somoano García O, Málaga Guerrero S, Riaño Galán I. Calidad de vida en niños y adolescentes con problemas crónicos de salud. Tipica, Boletín Electrónico de Salud Escolar 2010;6(2):250-6.

2. Mason J, Danby S. Children as experts in their lives: Child Inclusive Research. Child Ind Res 2011;4:185-9.

3. Shumaker S, Naughton $\mathrm{M}$. The international assesment of elath related quality of life: a theoretical perspective. En: Shumaker S, Berson R (ed). The international assessment of health related quality of life: Theory, translation, measurement and analysis. Oxford: Rapid Communications; 1995.

4. Bullinger, M. Quality of life: Definition, conceptualization and implications: a methologistis view. Theor Surg 1991;6: 143-8.

5. Urzúa, A.Calidad de vida relacionada a la salud: Elementos conceptuales. Rev Med Chile 2010; 138:358-65.

6. Guyatt GH, Ferrans CE, Halyard MY, Revicki DA, et al. Exploration of the Value of Health-Related Quality-ofLife information from Clinical Research and into Clinical Practice. Mayo Clinic Proc 2007;82(10):1229-39.

7. Rajmil JL, Roizen M, Urzúa A. Calidad de Vida y Salud en la Infancia y la Adolescencia. Boletín Electrónico de Salud Escolar 2010;6(2):244-9.

8. Rajmil L, Estrada MD, Herdmana M, Serra-Suttona V, et al. Calidad de vida relacionada con la salud (CVRS) en la infancia y la adolescencia: revisión de la bibliografía y de los instrumentos adaptados en España. Gac Sanit 2001;15 (Supl. 4):34-43.

9. Consiglio E, Belloso WH. Nuevos indicadores clínicos. La calidad de vida relacionada con la salud. Medicina (B. Aires) 2003; 63(2):172-8.

10. Pane S, Solans M, Gaite L, Serra-Sutton V, et al. Document d'Avaluació. Instrumentos de calidad de vida relacionada con la salud en la edad pediátrica. Revisión sistemática de la literatura: actualización. Barcelona: Agencia d'Avaluació de Tecnologia i Recerca Mèdiques, enero de 2006.

11. Mearin ML. Celiac disease among children and adolescents. Curr Probl Pediatr Adolesc Health Care 2007;37(3):86-105.

12. Olssoon C, Lyon P, Hörnell A, Ivarsson A, Sydner YM. Food that makes you different: The stigma experienced by adolescents with celiac disease. Qual Health Res 2009;19(7);976-84.

13. Roma E, Roubani A, Kolia E, Panayiotou J, et al. Dietary compliance and life style of children with coeliac disease. J Hum Nutri Diet 2010;23(2):176-82.

14. Hopman E, Koopman HM, Wit JM, Mearin ML. Dietary compliance and health-related quality of life in patients with coeliac disease. Eur J Gastroenterol Hepatol 2009;21(9):105661.

15. van Koppen EJ, Schweizer JJ, Csizmadia CG, Krom Y, et al. Long-term health and quality-of-Life. Consequences of mass screening for childhood celiac disease: A 10-Year Follow-up Study. Pediatrics 2009; 123(4): e582-8.

16. Van Doorn RK, Winkler LMF, Zwinderman KH, Mearin ML, Koopman HM. The CDDUX: A disease-specific healthrelated quality of life questionnaire for children with Celiac Disease. J Pediatr Gastroenterol Nutr 2008; 47(2):147-52.

17. Beaton D, Bombardier C, Guillemin F,Ferraz M. Guidelines for the process of cross-cultural adaptation of self-report measures. Spine (Phila Pa 1976) 2000;25(24):3186-91.

18. Pico M, Spirito MF. Adaptación transcultural del CDDUX: Versión española-argentina de un cuestionario de calidad de vida relacionada a la salud específico para niños con enfermedad celíaca. Medicina Infantil 2009;16:387-93.

19. Pico M, Spirito MF, Roizen M. Calidad de vida en niños y adolescentes con enfermedad celíaca: Versión argentina del cuestionario específico CDDUX. Acta Gastroenterol Latinoam 2012;42(1):12-9.

20. Solans M, Pane S, Estrada MD, Serra-Sutton V, et al. HealthRelated Quality of Life Measurement in Children and Adolescents: A Systematic Review of Generic and DiseaseSpecific Instruments. Value Health 2008:11(4):742-64.

21. Roizen M, Figueroa C, Salvia L y miembros del Comité de Calidad de Vida y Salud. Calidad de vida relacionada con la salud en niños con enfermedades crónicas: comparación de la visión de los niños, sus padres y sus médicos. Arch Argent Pediatr 2007;105(4):305-13.

22. Eiser C, Morse R. Can parents rate their child's health related quality of life? Results of a systematic review. Qual Life Res 2001;10(4): 347-57.

23. Janse AJ, Uiterwaal CS, Gemke RJ, Kimpen JL, Sinnema G. A difference in perception of quality of life in chronically ill children was found between parents and pediatrician. J Clin Epidemiol 2005;58(5):495-502.

24. Ellert U, Ravens-Sieberer U, Erhat M, Kurth BM. Health and Determinants of agreement between self-reported and parent-assessed quality of life for children in Germany. Results of the German Health Interview and Examination Survey for Children and Adolescents (KiGGS). Health Qual Life Outcomes 2011; 9:102. 
e26 / Arch Argent Pediatr 2014;112(1):e19-e25 / Artículo original

\section{Anexo 1. Formulario de implementación del cuestionario CDDUX-Versión Paciente}

Identificación del paciente:

Fecha:

Pregunta

Sí

No

¿Te demandó mucho tiempo contestar el cuestionario?

¿Te resultó molesto haberlo hecho?

¿Te hizo pensar en cosas que no habías pensado antes?

¿Te parece que ayuda a expresar cosas que de otra manera no

te hubieran preguntado?

¿Pensás que es importante que el médico sepa lo que

contestaste en el cuestionario para poder ayudarte?

¿Algún comentario, apreciación o sugerencia que quisiera decir respecto de la experiencia? 
Anexo 2. Formulario de implementación del cuestionario CDDUX-Versión Padre/Tutor

Identificación del paciente:

Fecha:

Pregunta

Sí

No

¿Le demandó mucho tiempo contestar el cuestionario?

¿Le resultó molesto haberlo hecho?

¿Le hizo pensar en cosas que no había pensado antes?

¿Le parece que ayuda a expresar cosas que de otra manera no

hubieran sido consideradas en la consulta?

¿Considera que es importante que el médico sepa los aspectos

que se abordan en el cuestionario para poder ayudar a su hijo/a?

¿Algún comentario, apreciación o sugerencia que quisiera decir respecto de la experiencia? 


\section{Anexo 3. Formulario de implementación del cuestionario CDDUX-Versión gastroenterólogo}

Fecha:

Identificación del paciente:

Identificación del médico:

¿Considera que la aplicación del CDDUX entorpeció el circuito de atención del paciente?

¿Afectó el desarrollo fluido de la consulta?

¿Los resultados del cuestionario brindaron información que le pareció importante?

A partir de estos resultados, ¿detectó algún aspecto que considera que necesita alguna acción?

Si la respuesta previa fue SÍ, ¿cuál/les de las siguientes acciones realizó?

- Indagar más profundamente el/los aspectos que observó negativos

- Derivar a ...................(nutricionista/ psicólogo/clínico/asistente social) para profundizar aspectos que observó problemáticos

- Otra acción:......................................................

¿Considera que este cuestionario puede ser incluido en forma sistemática en la consulta?

¿Algún comentario, apreciación o sugerencia que quisiera manifestar respecto de la experiencia? 